\title{
Overview of MIGS
}

\author{
Jing Wang and Keith Barton
}

The term, minimally- or micro-invasive glaucoma surgery (MIGS), first coined around 2008 (II Ahmed, personal communication) has entered common ophthalmic parlance and is playing an increasing role in the management of glaucoma patients. In common, the devices and procedures referred to, are safer, less tissue invasive and associated with faster recovery than traditional filtering surgery, such as trabeculectomy or aqueous shunt implantation [1]. While the term initially referred only to ab interno Schlemm's canal bypass stents such as the iStent, it has expanded, though with somewhat inconsistent adoption, both by clinicians and by the manufacturers, not all of whom are enthusiastic about applying the MIGS label to their device, to encompass both ab externo and ab interno canal procedures, suprachoroidal implants, external filtration devices and to some degree, even new types of cyclodestruction. On the horizon are also drug-eluting implants. The US Food and Drug Administration (USFDA) defines MIGS as devices or procedures that lower intra-ocular pressure (IOP) with either an ab interno or ab externo approach, associated with little or no scleral dissection and minimal or no conjunctival manipulation, though USFDA workshops and guidance have tended to consider only implantable devices $[2,3]$. This book covers the techniques that are most commonly regarded as eligible to sit under the MIGS umbrella, whether or not industry or clinicians prefer to call them MIGS. Others, such as the Ex-PRESS shunt (Alcon Laboratories, Inc., Fort Worth, Texas, USA), SOLX Gold Glaucoma Shunt (GGS, SOLX Ltd.,

\footnotetext{
J. Wang

Centre Universitaire d'ophtalmologie (CUO), Hôpital du Saint-Sacrement, CHU-Quebec, Québec, QC, Canada

Laval University, Québec, QC, Canada

K. Barton $(\bowtie)$

Glaucoma Service, Moorfields Eye Hospital, London, UK

Institute of Ophthalmology, University College London, London, UK

e-mail: keith@keithbarton.co.uk 
Waltham, MA, USA) and canaloplasty have some similarities to MIGS techniques and devices, but will not be covered in detail.

A number of MIGS devices and techniques have relatively modest efficacy but, potential utility in a very large group of glaucoma patients with disease that is insufficiently severe to justify the invasiveness of conventional filtration surgery and the consequent intensity of postoperative care, yet burdened with medication and the attendant side effects and compliance issues thereof. A simple additional technique at the time of cataract surgery could have significant quality of life benefits for a large number of these patients. On the other hand, some MIGS devices can potentially achieve efficacy approaching that of traditional filtering surgery and are appropriate in selected individuals when larger IOP reductions are required, the exception being cases where glaucoma is very advanced.

Irrespective of the modest efficacy of many MIGS devices and techniques, the favourable safety profile lowers the threshold for early glaucoma surgery, especially when combined with cataract surgery, potentially delaying the requirement for more invasive surgery and associated risks. The additional reduction in the medication burden has the potential to reduce intolerance, improve quality of life and lower the long-term cost of medication while improving adherence.

MIGS can be categorized according to the tissue they target (or bypass): trabecular meshwork (TM) MIGS, subconjunctival MIGS, suprachoroidal MIGS and newer cycloablation procedures. MIGS devices include iStent Trabecular MicroBypass Stent and iStent inject (Glaukos Corporation, San Clemente, CA, USA), Hydrus Microstent (Ivantis Inc., Irvine, CA, USA), the XEN Gel Implant (Allergan plc, Dublin, Ireland) and PRESERFLO (formerly InnFocus) MicroShunt (Santen Pharmaceutical Co. Ltd., Osaka, Japan) (Table 1.1). At present, as a result of the

Table 1.1 Procedures and implants that fall broadly within the minimally invasive category of glaucoma surgery though a number of those listed would not be typically described as MIGS

\begin{tabular}{|c|c|c|c|}
\hline Schlemm's canal & Subconjunctival & Suprachoroidal & Ciliary body coagulation \\
\hline $\begin{array}{l}\text { Stenting } \\
\text { iStent Trabecular } \\
\text { Micro-Bypass Stent } \\
\text { iStent inject } \\
\text { Hydrus Microstent }\end{array}$ & $\begin{array}{l}\text { Xen Gel Implant } \\
\text { PRESERFLO } \\
\text { MicroShunt }\end{array}$ & $\begin{array}{l}\text { iStent Supra } \\
\text { MINIject } \\
\text { (CyPass } \\
\text { Micro-Stent) }\end{array}$ & $\begin{array}{l}\text { High-Intensity Focused } \\
\text { Ultrasound cyclocoagulation } \\
\text { Micropulse diode laser } \\
\text { cyclophotocoagulation } \\
\text { Endocyclophotocoagulation }\end{array}$ \\
\hline $\begin{array}{l}\text { Cutting } \\
\text { Trabectome (Ab interno } \\
\text { trabeculotomy) } \\
\text { Gonioscopy-assisted } \\
\text { transluminal } \\
\text { trabeculotomy (GATT) } \\
\text { Excimer laser } \\
\text { trabeculostomy } \\
\text { Kahook Dual } \\
\text { Blade (KDB) }\end{array}$ & & & \\
\hline $\begin{array}{l}\text { Dilating } \\
\text { Ab interno } \\
\text { canaloplasty }(\mathrm{ABiC})\end{array}$ & & & \\
\hline
\end{tabular}


withdrawal of the CyPass Micro-Stent (Alcon Laboratories, Inc., Fort Worth, Texas, USA), there are no commercially available devices that drain to the supra-choroidal space, though others are in development.

While there are a number of pathways targeted by MIGS devices, most MIGS procedures in which a device is not implanted, are designed to eliminate trabecular meshwork resistance from the outflow pathway: $a b$ interno trabeculotomy (Trabectome; NeoMedix Corporation, San Juan Capistrano, CA, USA) and gonioscopic-assisted transluminal trabeculotomy (GATT). Newer surgical instruments such as the Kahook Dual Blade (New World Medical, Rancho Cucamonga, CA, USA) and TRAB360 (Sight Sciences Inc., Menlo Park, CA, USA) are designed for $a b$ interno removal of TM tissue to enhance physiological TM outflow system.

Ab interno canaloplasty (ABiC, Ellex Medical Pty Ltd., Adelaide, Australia) differs slightly in that it primarily dilates Schlemm's canal, although a small cut is made through trabecular meshwork to access the canal.

Concurrent with the appearance of the MIGS genre, a number of new cycloablation procedures have also appeared including micropulse diode laser trans-scleral cyclophotocoagulation (MicroPulse P3, IRIDEX IQ810 Laser System, Mountain View, CA, USA), applied externally via a new type of probe and High-Intensity Focused Ultrasound cyclocoagulation (EyeOP1 HIFU, EyeTechCare, Rillieux-laPape, France), applied externally but delivering a metered dose of ultrasound energy to the ciliary body. Endocylophotocoagulation (ECP), which was developed in the late 1990s, is analogous to conventional diode laser CPC, but applied via an $a b$ interno approach and could also be considered in this category.

\subsection{Trabecular Meshwork MIGS Devices and Techniques}

Trabecular meshwork (TM) MIGS procedures and devices are numerous. They aim to eliminate trabecular meshwork resistance in the normal physiological outflow pathway in patients with mild-to-moderate glaucoma and ocular hypertension (OHT). They are indicated in combination with cataract surgery. In patients with chronic primary angle closure, the TM outflow system has likely long-standing and irreversible damage; TM MIGS procedures or implants should be approached with caution as the drainage pathway created whether stent or trabeculotomy, may occlude with iris because of the narrow angle. In angle closure, these procedures should generally be considered only after cataract surgery and confirmation that the angle has widened sufficiently that the risk of occlusion is low. In patients with advanced glaucoma, where the maximum possible pressure lowering is often desirable in order to minimize the risk of disease progression, TM MIGS procedures are not ideal as there is an opportunity cost in not achieving IOP control with the first surgical procedure.

All TM MIGS procedures involve direct gonioscopic visualization during surgery. TM MIGS devices include iStent Trabecular Micro-Bypass Stent, iStent inject (Fig. 1.1) and Hydrus Microstent (Fig. 1.2) [4-6]. These three devices aim to enhance TM outflow by stenting the Schlemm's canal. iStent Trabecular MicroBypass Stent and iStent inject are manufactured from heparin-coated titanium. 

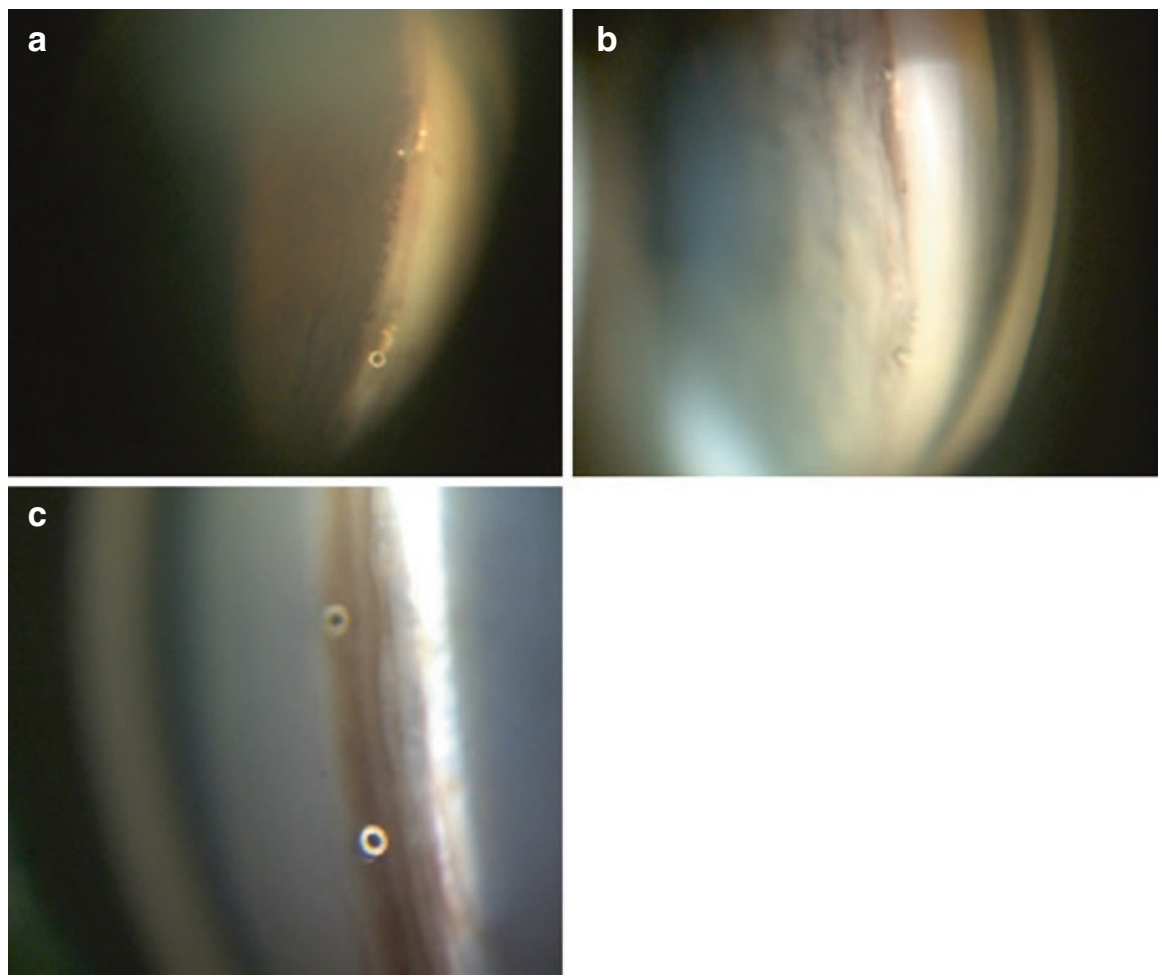

Fig. 1.1 Two iStent Trabecular Micro-Bypass Stents in the Schlemm's canal of two different patients (a and b) and two iStent inject implants in the trabecular meshwork (c). (Copyright Moorfields Eye Hospital and Keith Barton; reproduced with permission)
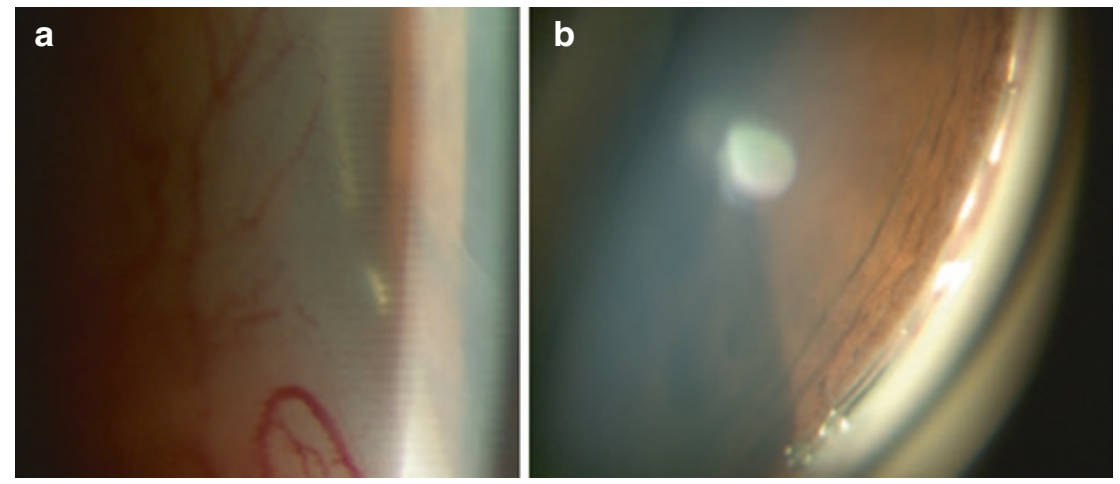

Fig. 1.2 The inlet of a Hydrus Microstent visible externally (a) and the Hydrus Microstent in the trabecular meshwork on gonioscopy (b). (Copyright Moorfields Eye Hospital and Keith Barton; reproduced with permission) 
iStent Trabecular Micro-Bypass Stent is a $1 \mathrm{~mm}$ long, L-shaped device with a $120 \mu \mathrm{m}$ lumen diameter. iStent inject is conically shaped, $360 \mu \mathrm{m}$ in length and $230 \mu \mathrm{m}$ at its largest diameter. The Hydrus Microstent is made of nitinol and is a crescent-shaped trabecular scaffold of $8 \mathrm{~mm}$ in length with a variable lumen diameter between 185 and $292 \mu \mathrm{m}$. Company-sponsored prospective randomized controlled trials have compared the effect of cataract surgery on IOP when combined with the iStent Trabecular Micro-Bypass Stent or the Hydrus Microstent to the effect of cataract surgery alone $[4,6]$. Both demonstrated a modest but more sustained IOP-lowering effect in the group receiving cataract surgery combined with the TM MIGS device 2 years after surgery. All three are USFDA approved, at the time of writing, for implantation at the time of cataract extraction, but not for standalone surgery. In Europe, they are licensed for both.

Other TM procedures such as ab interno trabeculotomy (AIT) or Trabectome, GATT, Kahook Dual Blade and TRAB360 cut rather than stent the TM to varying degrees. Trabectome is the earliest FDA-approved TM removal procedure. It has a disposable 19.5-gauge handpiece with irrigation, aspiration and electrocautery combined. The tip of the Trabectome removes TM tissue and coagulates at the same time. Trabectome surgery is either performed at the beginning of cataract surgery or as a stand-alone procedure [7]. The Kahook Dual Blade is a disposable knife designed to remove a strip of TM tissue via a temporal incision. With a single incision, the Kahook Dual Blade and Trabectome can remove up to $120^{\circ}$ of TM tissue, whereas GATT and TRAB360 (Sight Sciences, Menlo Park, CA, USA) can remove the entire TM tissue. GATT can be performed using either an illuminated microcatheter (iTrack, Ellex Medical Pty Ltd., Adelaide, Australia)—designed originally for ab externo canaloplasty procedure-or a 5-0 polypropylene or Nylon suture [8]. Under direct gonioscopic view, a micro vitreoretinal (MVR) blade is used to incise the TM wall, after which the catheter or suture is advanced to cannulate Schlemm's canal through the incision. Complete $360^{\circ}$ catheterization of Schlemm's canal may not be possible in all eyes. A prospective non-comparative case series has reported sustained IOP lowering for up to 2 years after GATT [9]. As $360^{\circ}$ trabeculotomy becomes a popular first-line intervention in primary congenital glaucoma, there has been some interest in treating juvenile open-angle glaucoma with GATT as a primary surgical option.

\subsection{Subconjunctival MIGS Devices}

The XEN Gel Implant (Allergan; formerly known as XEN Gel Stent, AqueSys Inc.) (Fig. 1.3) and PRESERFLO (formerly InnFocus) MicroShunt (Santen Pharmaceutical Co. Ltd.) (Fig. 1.4) are the two currently available subconjunctival MIGS devices $[10,11]$. The XEN Gel Implant is a soft porcine-derived collagen implant that is inserted, $a b$ interno, from the anterior chamber to subconjunctival space. Six millimetres long and with an internal diameter of $45 \mu \mathrm{m}$, the XEN is preloaded in an injector. Its major potential advantage over traditional filtering 

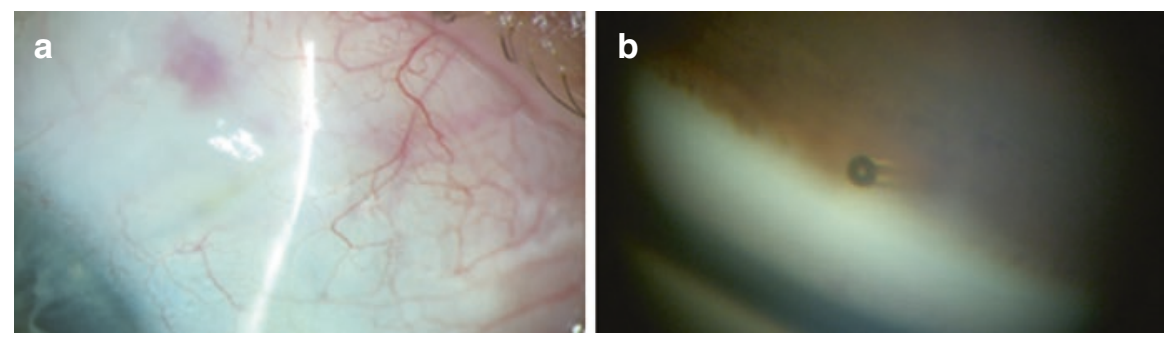

Fig. 1.3 XEN Gel Implant visible under the conjunctiva with a diffuse overlying drainage bleb (a) and the XEN Gel Implant visible in the anterior chamber (b). (Copyright Moorfields Eye Hospital and Keith Barton; reproduced with permission)
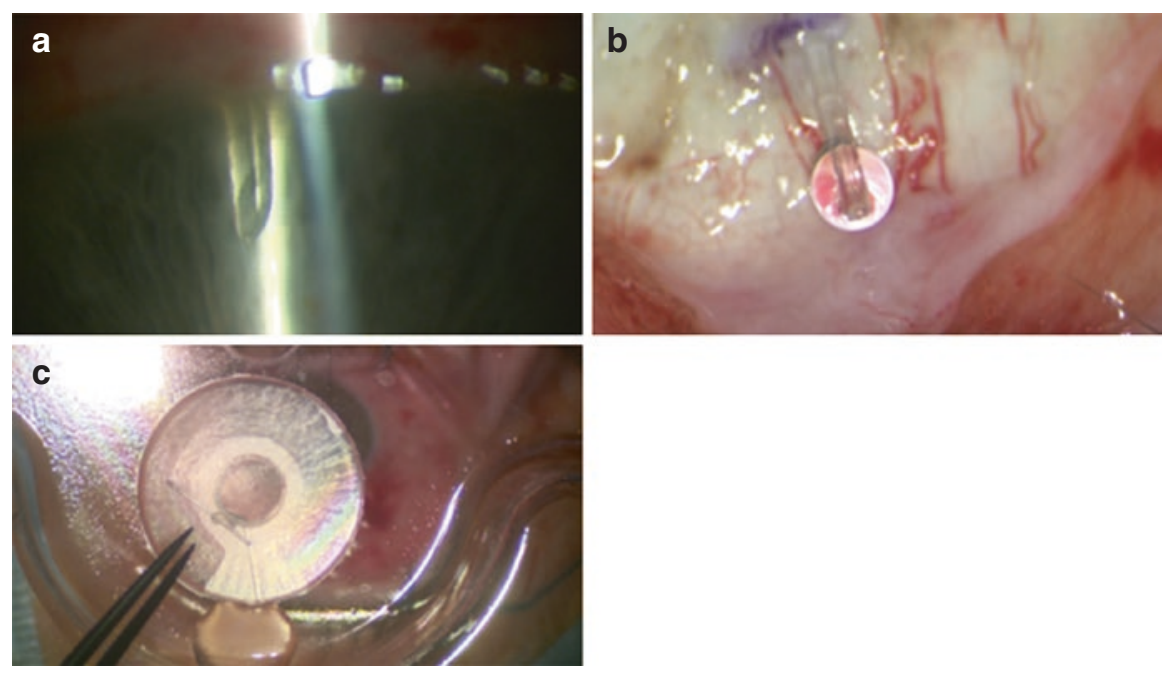

Fig. 1.4 The PRESERFLO MicroShunt in the anterior chamber (a), an external view showing aqueous drainage during implantation and before conjunctival closure (b) and the device prior to implantation (c). (Copyright Moorfields Eye Hospital and Keith Barton; reproduced with permission)

surgery is the avoidance of a conjunctival incision. However, the lack of conjunctival dissection requires precise placement of the XEN under the conjunctival tissue as the lumen of the XEN is easily blocked by Tenon's capsule. This explains a significantly higher rate of needling with the XEN [12]. Similar to the XEN Gel Implant, the PRESERFLO MicroShunt is also a tube that diverts aqueous humour from the anterior chamber to the subconjunctival space. The MicroShunt differs from the XEN in that it is implanted via an ab externo approach, necessitating conjunctival dissection. Unlike the XEN, the MicroShunt is of purely synthetic construction-poly(styrene-block-isobutylene-block-styrene) or SIBS. A randomized controlled trial comparing the MicroShunt with mitomycin C (MMC) to trabeculectomy with $\mathrm{MMC}$ for primary open angle glaucoma (POAG) is currently ongoing 
(ClinicalTrial: NCT01881425). This is currently the only MIGS device that has been compared to trabeculectomy in a randomized clinical trial.

The IOP-lowering efficacy of subconjunctival MIGS, in selected cases, appears to approach that of traditional filtering surgery, thereby offering the possibility that they might have utility in more advanced or normal pressure glaucoma. On the other hand, subconjunctival MIGS are bleb-forming procedures and serious bleb-related complications such as infection, leakage and implant exposure have been reported [13].

\subsection{Suprachoroidal MIGS Devices}

Until recently, CyPass Micro-Stent was the only available suprachoroidal MIGS. It is a fenestrated micro-stent of $6.35 \mathrm{~mm}$ long with an external diameter of $510 \mu \mathrm{m}$ and an internal diameter of $300 \mu \mathrm{m}$. It is made of a biocompatible polyamide material. The COMPASS trial is a randomized controlled trial comparing the effect of combined cataract surgery and CyPass insertion to cataract surgery alone in 505 POAG patients [14]. Two years after surgery, the IOP was lower on less medication in the group that underwent combined CyPass Micro-Stent implantation and cataract surgery than those that had cataract surgery alone. A prospective series of CyPass Micro-Stent implantation as a solo procedure in POAG patients with uncontrolled IOP demonstrated effective IOP lowering and avoided conventional filtering procedures in $83 \%$ of patients at 1 year follow-up [15]. After the COMPASS study was extended to 5 years after surgery (COMPASS XT), there was a significantly higher rate of endothelial cell loss in the combined CyPass Micro-Stent and cataract group compared to the cataract group alone. For this reason, the manufacturer (Alcon Laboratories, Inc., Fort Worth, Texas, USA) voluntarily withdrew the CyPass Micro-Stent from the market in August 2018, although it is estimated that there are currently around 33,000 implanted CyPass Micro-Stents in the world and managing the risk of endothelial loss may be an ongoing concern for several years after the withdrawal [16].

The iStent Supra (Glaukos) is a suprachoroidal stent made of polyethersulfone and heparin-coated titanium with a lumen diameter of $165 \mu \mathrm{m}$. The iStent Supra is not commercially available and there have been no prospective published efficacy studies at the time of writing.

\subsection{Cyclophotocoagulation (CPC) Procedures}

Cyclophotocoagulation procedures are also minimally invasive though they differ in that they reduce aqueous production by coagulating ciliary body tissue and are often not included within the MIGS genre.

Endocyclophotocoagulation (ECP) is an ab interno cycloablative procedure. An endoscopic camera equipped with an $810 \mathrm{~nm}$ diode laser probe in a single 18-20 gauge fibreoptic probe. The ciliary body epithelium is directly visualized during 
treatment; usually $240-300^{\circ}$ of ciliary body are treated with one incision. Two incisions are required for a full $360^{\circ}$ treatment [17]. There is no prospective randomized controlled trial on the efficacy of ECP. A case series comparing ECP combined with cataract extraction and cataract extraction alone found slightly lower IOP in the combined group. A retrospective case series comparing ECP with a second glaucoma drainage device (aqueous shunt) in patients with failed previous aqueous shunt surgery found similar IOP outcome at 1 year [18]. Post-operative complications of ECP include inflammation, hypotony, uncontrolled IOP, cystoid macular oedema $(10 \%)$ and phthisis. Intracameral triamcinolone is suggested to prevent fibrinous inflammation after ECP. Despite its ab interno approach, ECP theoretically can cause significant tissue damage and serious complications such as phthisis. Caution should therefore be taken in high-risk eyes.

Micropulse diode laser is a newer method of delivering diode laser to ocular tissue. Conventional laser application is continuous with a single pulse that lasts from 0.1 to $0.5 \mathrm{~s}$. In conventional diode cyclophotocoagulation, the duration of a single laser pulse is usually as long as few seconds. Micropulse mode laser delivers the energy in pulses with pre-set on and off periods. The off period is longer than the on period allowing the tissue to cool down and minimize damage. Micropulse laser has been used in the treatment of retinal diseases and glaucoma. In one prospective randomized series, micropulse cyclophotocoagulation is shown to be as efficient, resulting in similar IOP with less complications compared with conventional CPC.

\subsection{Overview Summary}

Subconjunctival drainage of aqueous humour has been the cornerstone of glaucoma surgery. MIGS devices targeting subconjunctival drainage achieve lower IOP than those targeting Schlemm's canal and suprachoroidal drainage, at the cost of possible bleb-related and higher hypotony-related complications. MIGS targeting the trabecular outflow system such as iStent Trabecular Micro-Bypass Stent or iStent inject, Hydrus Microstent and AIT are best suited for patients with moderate OHT or mild to moderate POAG requiring cataract surgery. The IOP-lowering effect of these trabecular devices is limited by episcleral venous pressure (EVP) which limits the maximal IOP reduction to the mid-teens. Subconjunctival draining devices (XEN Gel Implant or PRESERFLO MicroShunt) can be used as solo glaucoma procedure and have better potential to achieve single digit IOP levels. The long-term efficacy of sub-conjunctival MIGS is still unknown as there are few published data on these devices. They both require anti-metabolite (MMC) use as subconjunctival scarring is inevitable with the diversion of aqueous humour to the subconjunctival space. Suprachoroidal drainage devices aim at a potential space where IOP lowering is not limited by EVP and bleb formation is avoided. Scarring in the suprachoroidal space remains an issue. Suprachoroidal devices can potentially be used as an adjunct to traditional glaucoma surgery if further IOP-lowering is required. 


\section{References}

1. Saheb H, Ahmed II. Micro-invasive glaucoma surgery: current perspectives and future directions. Curr Opin Ophthalmol. 2012;23(2):96-104.

2. Premarket studies of implantable Minimally Invasive Glaucoma Surgical (MIGS) devices: Guidance for Industry and Food and Drug Administration Staff. [cited 2 November 2019]. https://www.fda.gov/media/90950/download.

3. Caprioli J, et al. Special commentary: supporting innovation for safe and effective minimally invasive glaucoma surgery: summary of a joint meeting of the American Glaucoma Society and the Food and Drug Administration, Washington, DC, February 26, 2014. Ophthalmology. 2015;122(9):1795-801.

4. Samuelson TW, et al. Randomized evaluation of the trabecular micro-bypass stent with phacoemulsification in patients with glaucoma and cataract. Ophthalmology. 2011;118(3):459-67.

5. Camras LJ, et al. A novel Schlemm's Canal scaffold increases outflow facility in a human anterior segment perfusion model. Invest Ophthalmol Vis Sci. 2012;53(10):6115-21.

6. Pfeiffer N, et al. A randomized trial of a Schlemm's canal microstent with phacoemulsification for reducing intraocular pressure in open-angle glaucoma. Ophthalmology. 2015;122(7):1283-93.

7. Minckler D, et al. Clinical results with the Trabectome, a novel surgical device for treatment of open-angle glaucoma. Trans Am Ophthalmol Soc. 2006;104:40-50.

8. Grover DS, et al. Gonioscopy-assisted transluminal trabeculotomy, ab interno trabeculotomy: technique report and preliminary results. Ophthalmology. 2014;121(4):855-61.

9. Grover DS, et al. Gonioscopy-assisted transluminal trabeculotomy: an ab interno circumferential trabeculotomy: 24 months follow-up. J Glaucoma. 2018;27(5):393-401.

10. Lewis RA. Ab interno approach to the subconjunctival space using a collagen glaucoma stent. J Cataract Refract Surg. 2014;40(8):1301-6.

11. Batlle JF, et al. Three-year follow-up of a novel aqueous humor MicroShunt. J Glaucoma. 2016;25(2):e58-65.

12. Schlenker MB, et al. Efficacy, safety, and risk factors for failure of standalone ab interno gelatin Microstent implantation versus standalone trabeculectomy. Ophthalmology. 2017;124(11):1579-88.

13. Kerr NM, et al. Ab interno gel implant-associated bleb-related infection. Am J Ophthalmol. 2018;189:96-101.

14. Vold S, et al. Two-year COMPASS trial results: supraciliary microstenting with phacoemulsification in patients with open-angle glaucoma and cataracts. Ophthalmology. 2016;123(10):2103-12.

15. Garcia-Feijoo J, et al. Supraciliary micro-stent implantation for open-angle glaucoma failing topical therapy: 1-year results of a multicenter study. Am J Ophthalmol. 2015;159(6):1075-1081.e1.

16. [cited 2 November 2019]. https://www.alcon.com/cypass-recall-information.

17. Kahook MY, Lathrop KL, Noecker RJ. One-site versus two-site endoscopic cyclophotocoagulation. J Glaucoma. 2007;16(6):527-30.

18. Murakami Y, et al. Endoscopic cyclophotocoagulation versus second glaucoma drainage device after prior aqueous tube shunt surgery. Clin Exp Ophthalmol. 2017;45:241-6. 
Open Access This chapter is licensed under the terms of the Creative Commons Attribution 4.0 International License (http://creativecommons.org/licenses/by/4.0/), which permits use, sharing, adaptation, distribution and reproduction in any medium or format, as long as you give appropriate credit to the original author(s) and the source, provide a link to the Creative Commons license and indicate if changes were made.

The images or other third party material in this chapter are included in the chapter's Creative Commons license, unless indicated otherwise in a credit line to the material. If material is not included in the chapter's Creative Commons license and your intended use is not permitted by statutory regulation or exceeds the permitted use, you will need to obtain permission directly from the copyright holder. 